\title{
Angioarchitecture of Brain AVM Determines the Presentation with Seizures: Proposed Scoring System
}

\author{
J.J.S. Shankar, R.J. Menezes, B. Pohlmann-Eden, C. Wallace, K. terBrugge, and T. Krings
}

\begin{abstract}
BACKGROUND AND PURPOSE: Seizures are a common presentation in patients with brain arteriovenous malformations, but the pathogenesis of seizures remains poorly understood. The purpose of our study was to analyze various morphologic and hemodynamic characteristics of unruptured BAVMs presenting primarily with seizures and, on the basis of these, to propose a scoring system to predict seizures in patients with BAVMs.
\end{abstract}

MATERIALS AND METHODS: In a retrospective review of our BAVM data base from 2000 to 2009, after excluding patients with ruptured BAVMs, we classified patients into 2 groups, those with and without seizures at presentation. Clinical, angiographic, and hemodynamic characteristics on cerebral angiograms of all these patients were studied. The association between various angioarchitectural variables and seizure presentation was examined, and these results were used to guide the development of criteria to predict presentation with seizures.

RESULTS: Of 1299 patients in our data base, we finally analyzed 33 patients with unruptured BAVMs with seizures and 45 patients with unruptured AVMs without seizures. Location, fistulous component in the nidus, venous outflow stenosis, and the presence of a long pial course of the draining vein were identified as the strongest predictors of seizures. The proposed scoring system had good predictability of presentation with seizures.

CONCLUSIONS: Specific angioarchitectural characteristics of unruptured BAVMs may predict occurrence of seizures and may help in targeted treatment.

ABBREVIATIONS: $\mathrm{ACA}=$ anterior cerebral artery; $\mathrm{BAVM}=$ brain arteriovenous malformation; $\mathrm{Cl}=$ confidence interval; $\mathrm{OR}=$ odds ratio; $\mathrm{PCA}=$ posterior cerebral artery

B rain arteriovenous malformations are presumably congenital vascular malformations that may present clinically with a variety of symptoms, including hemorrhage, seizures, headaches, progressive neurologic deficits, and intellectual deterioration. Approximately one-third to one-half of patients with newly diagnosed previously unruptured BAVMs present with seizures. ${ }^{1,2}$ One percent of unprovoked first seizures are caused by BAVMs. ${ }^{3}$ The etiology of seizures in BAVMs remains poorly understood and cannot be explained easily by their presence alone because similar-appearing BAVMs can present either with or without seizures. While seizure manifestation in previously ruptured

Received March 29, 2012; accepted after revision July 27.

From Toronto Western Hospital, Department of Medical Imaging, Division of Neuroradiology (J.J.S.S., R.J.M., K.t.B., T.K.) and Department of Neurosurgery (C.W.) Toronto, Ontario, Canada; and Department of Diagnostic Imaging, Division of Neuroradiology (J.J.S.S.) and Department of Neurology (B.P.-E.), QE II Hospital, Halifax, Nova Scotia, Canada.

Please address correspondence to Jai Jai Shiva Shankar, MD, Department of Diagnostic Imaging, Division of Neuroradiology, QE II Hospital, 5743 Southwood Dr, Halifax, NS, Canada B3H IE6; e-mail: shivajai1@rediffmail.com

http://dx.doi.org/10.3174/ajnr.A3361
BAVMs can be explained by defects and gliosis secondary to the intracerebral hemorrhage, ${ }^{2,4}$ seizure presentation in previously unruptured BAVMs is more difficult to explain. Neocortical localization of the BAVMs, especially in the frontal and temporal lobes, has been reported to be associated with seizure presentation. ${ }^{5,6}$ In addition, mass effect of the BAVM nidus or associated venous pouches ${ }^{7}$ may be responsible for triggering seizure activity.

Whether hemodynamic alterations caused by the often-complex angioarchitecture of BAVMs play a role in seizure manifestation has been a matter of debate. Large BAVM size and high flow through the arterial feeding vessels of the BAVMs were related to seizure susceptibility on the basis of the assumption that the large nidus creates a low-resistance vascular bed redirecting blood away from the surrounding brain tissue, thus creating local hypoxemia in the adjacent brain tissue, which, in turn, may trigger gliosis and seizure activity. This pathomechanism was referred to as "arterial steal." ${ }^{\text {-10 }}$ However, the actual existence of steal has been questioned because it has been difficult to demonstrate quantitatively. ${ }^{7,11,12}$ Others have raised the possibility that seizures are 
more often related to a disrupted venous outflow pattern than to an inadequacy of arterial blood supply. ${ }^{13-17}$

There are only a few studies that have examined the association between individual angioarchitectural characteristics of the BAVM and seizure presentation. ${ }^{3,18}$ Turjman et al, ${ }^{2}$ in their study of 100 consecutive patients with seizures, and Santos et al, ${ }^{18}$ in their study of 170 patients with BAVM, found multiple morphologic features of BAVM associated with seizures. However, these studies lacked a thorough analysis of the hemodynamic characteristics of the BAVM. Moreover, they did not incorporate a control group to compare these features in patients with BAVM who did not present with seizures. We, therefore, analyzed multiple morphologic and hemodynamic characteristics of the BAVMs in a consecutive series of patients, for whom high-resolution digital subtraction angiography existed, and we correlated our findings to the patients' clinical status (ie, whether seizures were present). On the basis of the angioarchitectural characteristics of our patients with seizures, we would like to propose a grading system that might be able to identify those patients with BAVMs who may be more prone to single seizures or to develop epilepsy. In addition, identification of specific angioarchitectural features that are more often associated with seizures may shed light on the complex pathophysiology of seizure occurrence in patients with unruptured BAVMs and may, thereby, act as therapeutic targets for either surgery or embolization. The proposed scoring system may help us in predicting seizures in incidentally found BAVMs on the basis of their angioarchitectural features.

\section{MATERIALS AND METHODS}

Institutional review board approval was obtained with a waiver of informed consent for this retrospective study. Patients were selected from a single-institution data base consisting of 1299 BAVMs presenting between June 1984 and January 2010. The data base has been collected prospectively since 1989 by a team of neuroradiologists and neurosurgeons in a multidisciplinary clinic. The evaluations in the data base include a detailed medical history, full neurologic examination, and imaging findings. In this study, we only included those patients who were entered since 2000 and in whom digital subtraction angiography was performed before any form of treatment. The PACS in our institute was only installed in the 2000, so we had to exclude patients who were imaged before that year. Because cerebral hemorrhage can lead to tissue damage and subsequently cause seizures, we excluded all patients with BAVMs with a history of cerebral hemorrhage. We classified the remainder of patients with BAVMs into 2 groups: those with seizures and those without seizures at presentation. The patients with BAVMs with no seizure at presentation formed our control group (Fig 1). The diagnosis of the seizures in these patients was made by our clinical team on the basis of the clinical history and electrophysiologic examination. We did not further subclassify the type of seizures in our patients because the number of patients in each subcategory was very small for any meaningful conclusion. We retrospectively studied the clinical characteristics and cerebral angiograms of these patients as indicated below.

\section{Image Acquisition and Analysis}

Only patients who underwent complete (bilateral internal carotid arteries, at least 1 vertebral artery, and at least the ipsilateral external carotid artery) biplane DSA during their initial work-up were included in this study. Supply from the external carotid artery may represent features of arterial steal/ischemia. Images were acquired at a rate of 3 frames per second with contrast-injection rates of $4 \mathrm{~mL} / \mathrm{s}$ for a total of $8 \mathrm{~mL}$, until the late venous phase. The different angioarchitectural characteristics of the BAVMs were analyzed on the basis of the anatomic characteristics such as location and dimensions of the BAVM and the hemodynamic characteristics, such as features of high flow with potential steal and features of venous outflow obstruction and congestion (Table 1). Most of the characteristics (venous and arterial traits, location, and size) were selected because they may be related to the likelihood of seizure occurrence by reflecting the variations in the hemodynamic status of the BAVM or factors of cortical irritation.

The BAVMs were classified as superficially located when they were supplied by the cortical branches of the ACA, MCA, or PCA. They were classified as deep when they were supplied by the lenticulostriate or thalamostriate arteries and had no component that reached the cortical surface. The measurements of the BAVM were obtained by taking into consideration the magnification factor of our angiographic equipment. "Arterial dilation" was defined as at least a 50\% increase in the diameter in comparison with the arteries in the same region with no contribution to the BAVM. "Fistulous component" of the nidus was defined as a direct transition of the artery into the vein with no intervening nidus network, with a shorter transit time compared with the rest of the BAVM (Fig 2B, -C). "Pial recruitment" or "perinidal angiogenesis" was identified as an indirect supply to the peripheral part of the BAVM from the branches of the arteries other than the primary arterial supply to the BAVM (Fig $2 B,-C$ ). "Pseudophlebitic pattern" was defined as presence of tortuous engorged collateral veins identified on the venous phase of the brain circulation with 
Table 1: Angioarchitectural features with significant association of seizure presentation

\begin{tabular}{lc}
\multicolumn{1}{c}{$\begin{array}{c}\text { BAVM Angioarchitectural } \\
\text { Characteristics }\end{array}$} & $\begin{array}{c}\text { Association with Seizures } \\
(\boldsymbol{P} \text { Value })\end{array}$ \\
\hline Indicators of high flow with potential steal & .0715 \\
Significant arterial dilation & .0112 \\
Pial recruitment/perinidal angiogenesis & .0013 \\
Fistulous component of nidus & .0067 \\
Intranidal aneurysm & \\
Indicators of venous outflow obstruction and congestion & $<.0001$ \\
Pial long draining vein & .0233 \\
Pseudophlebitic pattern & .0003 \\
Venous outflow restriction draining vein & .0213 \\
Venous ectasia & \\
\hline
\end{tabular}

delay in the circulation time (Fig 3B). ${ }^{19}$ The delay in the circulation time was assessed qualitatively as described by Willinsky et al. ${ }^{19}$ A "venous outflow stenosis" (Fig 3C) was defined as a reduction of $50 \%$ or more of the vein diameter, ${ }^{20}$ and "venous ectasia" (Fig 3D), as a markedly ectatic vein. ${ }^{21}$ "Long course of the draining vein" was defined as longer than a 3 -cm superficial course of the draining vein.

\section{Statistical Methods}

For each angioarchitectural characteristic, sensitivity, specificity, negative predictive value, positive predictive value, and corresponding 95\% confidence intervals with bootstrap methods were calculated for diagnosis of seizures. $\chi^{2}$ and Fisher exact tests were used to examine the association between the characteristics and outcome, while unadjusted logistic regression analysis and bootstrap methods were used to determine the nature and strength of the association with seizures.

Derivation of criteria for predicting seizures was affected by sample size, which limited the number of characteristics that could be examined simultaneously. Multivariate analysis was restricted to the 3 strongest predictors from univariate analysis so as to maintain an even per variable ratio of approximately $10 .{ }^{22} \mathrm{Mul}-$ tivariate logistic regression was attempted after testing for multicollinearity among the selected variables by measuring the variation inflation factor. The number of patients who fit each combination of the 3 strongest predictors was determined, and diagnostic performance criteria of each combination and the corresponding 95\% confidence interval were calculated with bootstrap methods. We examined the overall scoring system by using receiver operating characteristics analysis, calculating the area under the curve and corresponding 95\% confidence interval. For all analyses, a $P$ value $<.05$ was considered an indicator of a statistically significant association.

\section{RESULTS}

The distribution of the patients in our study is shown in Fig 1.

Fifteen patients from the BAVM with seizures group and 21 patients with BAVM without seizures could not be analyzed because the available DSA was either incomplete or all information required for the angioarchitectural classification was not available. Final analysis included 33 patients (male/female ratio, 21:12; mean age, 33 years; range, 9-66 years) with BAVM with seizures without intracranial hemorrhages and 45 patients (male/female ratio, 16:29; mean age, 34 years; range, 7-65 years) with BAVMs and without seizures and without intracranial hemorrhages.

Phi correlation analysis showed that there was no substantial correlation between the different investigated variables. Only venous ectasia and fistulous component showed a moderate level of correlation (correlation coefficient, 0.620).

The angioarchitectural features with significant association with the presence of seizures are listed in Table 1.

The association between the various angiographic characteristics with the occurrence of seizures is summarized in Table 2. Results indicated that all investigated angiographic characteristics were significantly associated with outcome, the strongest one with the long course of the pial draining vein, based on its odds ratio.

Size. BAVMs with a nidus size larger than $3 \mathrm{~cm}(n=33)$ presented significantly more often with seizures (20/33) compared with patients with BAVMs smaller than $3 \mathrm{~cm}(n=45,13 / 45)(P=.005)$.

Location. To evaluate the relationship between the location of the BAVM and seizure occurrence, we used 3 different approaches: superficial versus deep location, the feeding artery (MCA, ACA, PCA, perforators), and the lobes where the BAVM was located, taking into consideration that these 3 features are interdependent.

Superficial-versus-Deep Location. There was no significant influence of superficial-versus-deep location on the occurrence of seizures $(P=.10)$, presumably due to the small number of patients with deep BAVMs (11 deep versus 67 with superficial locations). However, seizures occurred in approximately $46 \%$ of the "superficial" group $(n=67,31 / 67)$ and in only 2 of the 11 patients with a "deep" location (18\%). Given the asymmetric distribution of deep-versus-superficial lesions, we did not pursue this evaluation further.

Feeding Artery. The feeding artery of the BAVM had a weak correlation with seizure occurrence only when we evaluated MCA involvement; if the MCA was not the feeder $(n=22)$, seizures occurred in only $13.6 \%(3 / 22)$, whereas in patients in whom the MCA was a feeder $(n=56)$, seizures were present in $53.6 \%$ (30/ 56) $\left(\chi^{2}=0.002\right)$.

Lobe. The frontal, temporal, and parietal locations of the BAVMs were significantly associated with the occurrence of seizures with an odds ratio of $5.00(95 \% \mathrm{CI}, 1.31-19.07)\left(P=.015, \chi^{2}\right)$. For subsequent analyses, we chose, therefore, "lobe" as a factor that may determine presentation with seizures. Individual analyses for every single lobe and for different permutations of lobes (frontal and temporal, temporal and parietal, and so forth) were also performed; however, these did not demonstrate a similar high OR.

Arterial Features. As seen in Table 2, three arterial features (fistulous component, arterial dilation, and perinidal angiogenesis) were evaluated. These 3 features indicate high flow throughput of the AVM with subsequent perinidal hypoxemia. Of these 3 fea- 


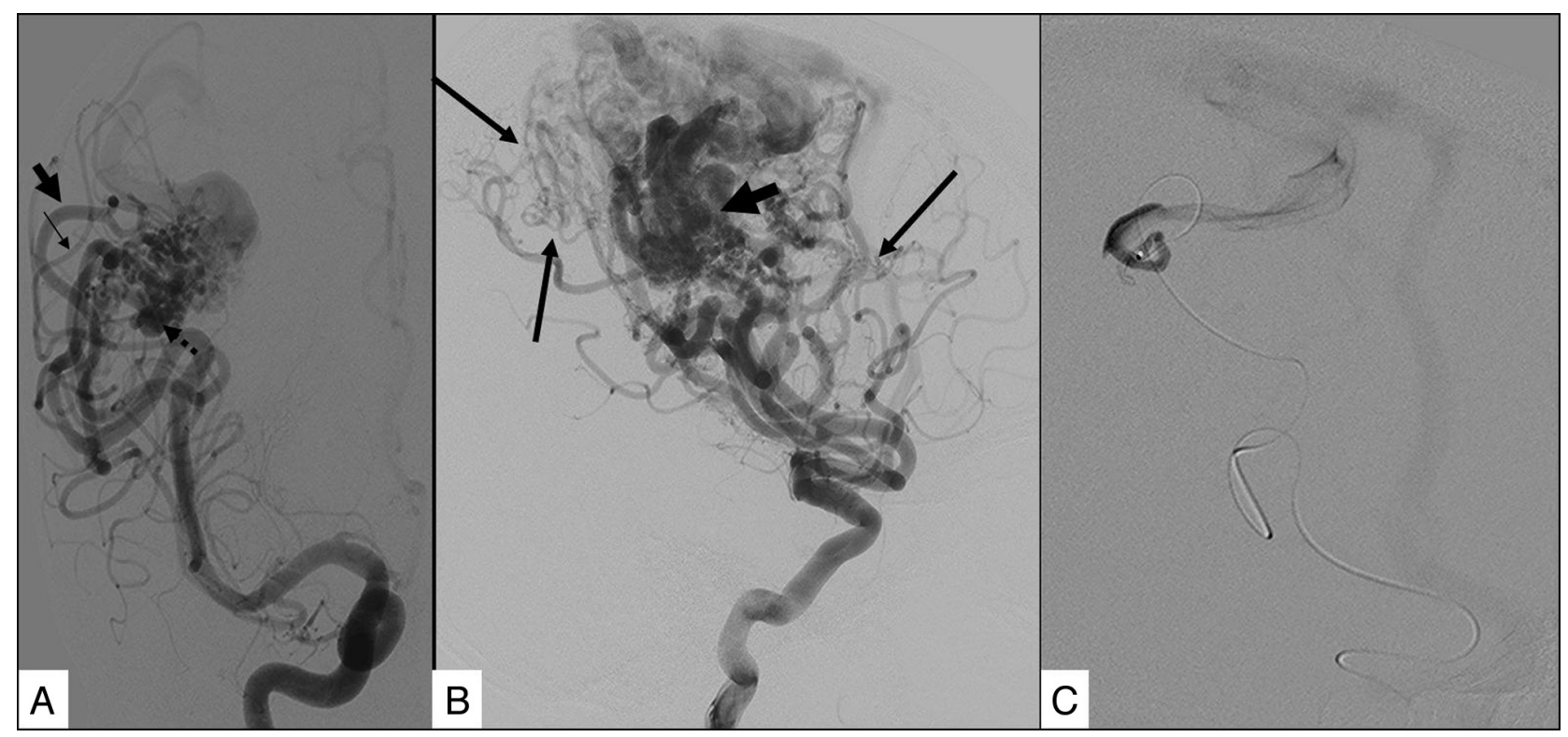

FIG 2. Features showing indicators of high flow with potential steal. A, Right frontal BAVM shows arterial dilation of the feeding artery (arrowhead) compared with a normal middle cerebral artery branch (small arrow) and an intranidal aneurysm (broken arrow). B, Frontoparietal BAVM shows perinidal angiogenesis (long arrows) and a fistulous component of the nidus (bold arrow). The fistulous component was further confirmed on selective microcatheter angiography (C).

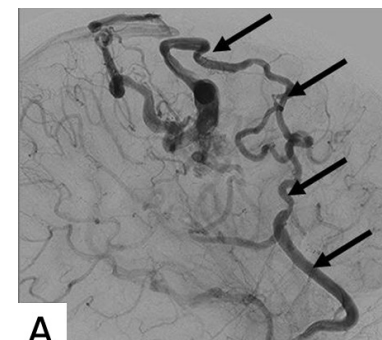

A
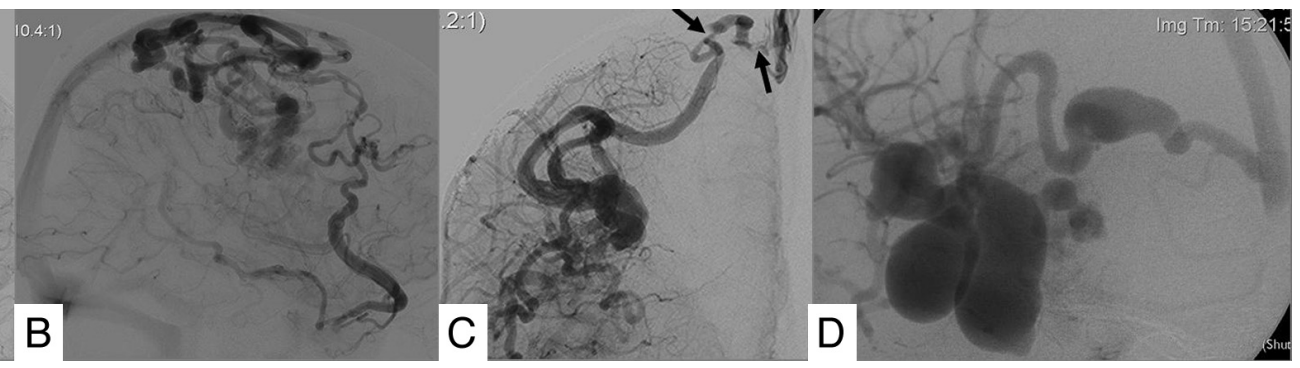

FIG 3. Features showing indicators of venous outflow obstruction and congestion. The venous phase of a frontoparietal BAVM shows the long pial course of the draining vein (arrows, $A$ ) and a pseudophlebitic pattern (B). The venous phase of a different BAVM shows venous outflow restriction of the draining vein (arrows, C) and venous ectasia (D).

Table 2: Various angioarchitectural characteristics and their association with the seizure presentation

\begin{tabular}{|c|c|c|c|c|c|c|}
\hline $\begin{array}{l}\text { Angiographic } \\
\text { Characteristics }\end{array}$ & 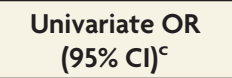 & $\begin{array}{c}\text { Multivariate OR } \\
\qquad(95 \% \mathrm{Cl})^{c}\end{array}$ & Sensitivity ${ }^{a}$ & Specificity $^{a}$ & $\mathrm{NPV}^{\mathrm{a}}$ & PPV $^{\mathbf{a}}$ \\
\hline Nidus size $>3 \mathrm{~cm}$ & 3.79 (1.47-10.86) & - & $61 \%(42-79)$ & $71 \%(58-82)$ & $71 \%(58-84)$ & $61 \%(46-79)$ \\
\hline Frontal, parietal, temporal & $5.00(1.50-19.07)$ & $4.52(0.95-21.47)$ & $91 \%(82-100)$ & $33 \%(20-47)$ & $83 \%(67-94)$ & $50 \%(38-62)$ \\
\hline Arterial dilation & $N A^{b}$ & - & $100 \%$ (NA) & $22 \%(11-36)$ & $100 \%$ (NA) & $49 \%(37-60)$ \\
\hline Perinidal angiogenesis & $3.71(1.47-12.05)$ & - & $70 \%(53-83)$ & $61 \%(48-75)$ & $75 \%(61-86)$ & $55 \%(40-71)$ \\
\hline Fistulous component & $4.11(1.45-13.20)$ & - & $75 \%(59-91)$ & $58 \%(44-71)$ & $76 \%(62-91)$ & $56 \%(42-70)$ \\
\hline Pial long draining vein & $14.86(5.67-66.09)$ & $5.71(1.32-24.65)$ & $79 \%(64-91)$ & $80 \%$ (69-91) & $84 \%(72-93)$ & $74 \%(60-89)$ \\
\hline Pseudophlebitic & 3.08 (1.21-9.25) & - & $61 \%(46-76)$ & $67 \%(53-80)$ & $70 \%(56-81)$ & $57 \%(40-74)$ \\
\hline Venous outflow stenosis & $6.50(2.22-32.49)$ & $6.71(1.99-22.56)$ & $50 \%(34-69)$ & $87 \%(76-96)$ & $71 \%(58-82)$ & $73 \%(55-91)$ \\
\hline Venous ectasia & $3.00(1.05-13.75)$ & - & $82 \%(70-94)$ & $40 \%(27-56)$ & $75 \%(58-92)$ & $50 \%(37-61)$ \\
\hline
\end{tabular}

Note:-NPV indicates negative predictive value; PPV, positive predictive value; NA, not applicable.

${ }^{a}$ Numbers in parentheses correspond to $95 \%$ confidence intervals for that diagnostic performance measure.

${ }^{b}$ No OR can be given for arterial dilation because zero patients with arterial dilation had no seizure presentation.

c All confidence intervals were generated via bootstrapping, except for those from multivariate analysis.

tures, the presence of a fistulous component had the strongest association with seizure occurrence and was, therefore, chosen for subsequent scoring: In 43 patients with fistulous components, 24 (56\%) presented with seizures, whereas in 34 patients without a fistulous component, only 8 (23.5\%) presented with seizures.

Venous Features. Of the 4 investigated venous features (long pial course of the draining vein, pseudophlebitic pattern, venous out- flow stenosis, and venous ectasia), 2 (long pial course of the draining vein and venous outflow restriction) demonstrated an OR of $>4$ (Table 2). The long pial course of the draining vein was the most significant marker for the occurrence of seizures: It was present in 35 patients, 26 of whom (74.3\%) presented with seizures. If the long pial course of the draining vein was not present ( $n=43$ patients), seizures were only present in 7 patients 


\begin{tabular}{lc}
\hline \multicolumn{1}{c}{ Parameter } & Score \\
\hline Venous outflow restriction & 1 \\
Location & 1 \\
Long pial draining vein & 1 \\
\hline
\end{tabular}

(16.3\%), indicating a high predictive value of this angioarchitectural feature in our patient cohort. We showed that the other 3 investigated factors (venous ectasia, venous outflow stenosis, and pseudophlebitic pattern) were not related to the long pial course of the draining vein and demonstrated venous characteristics that indicated high inflow and reduced outflow out of the venous system. The venous outflow stenosis was the second best predictor of seizure presentation. In 22 patients with this feature, 16 (73\%) presented with seizures. If it was not present ( $n=55$ patients), seizures were only present in 16 patients (29\%), indicating a high predictive value of this angioarchitectural feature in our patient cohort.

Multivariate analysis of the 3 strongest predictors from univariate analysis (venous outflow stenosis, long pial draining vein, and location of BAVM) showed high ORs of 6.71, 5.71, and 4.52, respectively.

\section{Proposed Scoring}

We included the 3 strongest predictors to establish a grading scale (Table 3). Venous outflow stenosis, long pial draining vein, and location of BAVM were given a score of 1 . This resulted in a scoring system of $0-3$ points.

This scoring system was a predictive model for our individual patients as outlined in Table 4. The area under the curve for this variable was 0.841 (95\% CI, 0.749-0.933) (Fig 4). The score of 0 had very high sensitivity and negative predictive value, whereas the score of 3 had a high specificity and positive predictive value in our group of patients. A threshold of 2 showed very good overall diagnostic performance.

\section{DISCUSSION}

Crawford et $\mathrm{al}^{23}$ studied various clinical factors such as age of the patient, presence of hemorrhage, and other neurologic symptoms predisposing to the development of seizures in patients with a BAVM. They found that the younger the patient was at diagnosis, the more likely he or she was to develop seizures on follow-up. Patients with hemorrhage had the greatest risk of developing seizures in their series. Only a few articles have exclusively studied the angioarchitectural characteristics of BAVMs associated with clinical presentation of seizures. ${ }^{3,18}$ Our experience showed that certain angioarchitectural characteristics are more often associated with seizure presentation. These include cortical location; features indicative of high flow such as the presence of a fistulous component in the nidus and perinidal angiogenesis; and features indicative of venous congestion, such as a long pial course of the draining vein, pseudophlebitic pattern, and venous outflow stenosis.

To identify angioarchitectural characteristics primarily related to seizures, one must study patients who present primarily with seizures without associated cerebral hemorrhage. It is also important to study whether these angiographic characteristics are un- common in patients without seizures. This research will help to identify the clinical presentation of seizures based solely on angioarchitecture.

We found multiple significant factors concerning the relationship of BAVMs to the occurrence of seizures, including size, location, and arterial and venous features.

Size. Size was significantly associated with the occurrence of seizures. Other studies have shown that large and medium nidus sizes (3-6 cm and $>6 \mathrm{~cm}$, respectively) were positively correlated with seizure occurrence, whereas small nidus size $(<3 \mathrm{~cm})$ was negatively correlated with presentation with seizures. ${ }^{18}$ A large BAVM may be more likely to present with seizures because the overall "sump effect" of the BAVM may be more prominent, compared with a small BAVM. Seizure occurrence may also be related to the larger volume of tissue in the circumferential vicinity of the BAVM, with a higher likelihood of affecting a potentially ictogenic zone, or it may be due to a more widespread hypoxemia. Holohemispheric brain BAVMs (so-called cerebral proliferative angiopathy) typically present with seizures. Perfusion studies performed in patients with cerebral proliferative angiopathy were able to demonstrate a widespread hypoperfusion distant from affected brain tissue, indicating hypoxemia in previously healthy brain tissue with subsequent gliosis. ${ }^{24}$

Location. We presume that frontal, temporal, or parietal location of the BAVM compared with deep-seated, infratentorial, or occipital BAVMs is associated with seizure occurrence because some type of cortical involvement is necessary to produce seizures. Deeply located BAVMs may present with seizure due to the interference of the venous drainage of mesial temporal lobe structures. Because the MCA is involved in the supply of frontal, parietal, and temporal lobes, the MCA feeder is also significantly associated with seizures.

Similar results have been shown in other studies. Frontal and parietal locations as well as feeding arteries from the MCA have been shown to be positively correlated with seizure occurrence, while the correlation between the occipital and cerebellar regions and deep location have been shown to be negative. ${ }^{5,6,18}$

Arterial Flow. Factors related to high flow such as dilation of the feeding artery and a fistulous component in the nidus can give rise to relative arterial functional steal in the region of the nidus. This has been described in the literature in the past ${ }^{8-10}$ and has been demonstrated in perfusion studies. ${ }^{25,26}$ This arterial steal with subsequent local hypoxemia may lead to an increased perinidal angiogenesis with blood being rerouted from the normal brain tissue due to the sump effect of the BAVM. Hypoxemia leads to an increased activity of the vascular endothelial growth factor triggered by the hypoxemia inducible factor ( $a$ and $b$ ). The subsequent perinidal angiogenesis, in turn, extends the area of functional arterial steal to the adjacent cortex, resulting in a larger area of cortex being hypoxemic. Chronic hypoxemia can lead to gliosis, which, in turn, may trigger epileptic activity. ${ }^{7,11,12}$ The fistula may also be a marker for greater venous hypertension due to greater bulk flow into the draining vein.

Venous Congestion. Venous congestion of normal brain tissue can be due to an increased inflow into the venous system (such as 
Table 4: Diagnostic performance of the scoring system ${ }^{\text {a }}$

\begin{tabular}{|c|c|c|c|c|c|c|}
\hline \multirow[b]{2}{*}{ Score } & \multicolumn{2}{|c|}{ Seizures } & \multirow[b]{2}{*}{ Sensitivity } & \multirow[b]{2}{*}{ Specificity } & \multirow[b]{2}{*}{ NPV } & \multirow[b]{2}{*}{ PPV } \\
\hline & No $(n=45)$ & Yes $(n=33)$ & & & & \\
\hline 0 & 13 & 1 & $97 \%$ & $29 \%$ & $93 \%$ & $49 \%$ \\
\hline$\geq 1$ & 32 & 31 & & & & \\
\hline$<2$ & 39 & 11 & $66 \%$ & $87 \%$ & $78 \%$ & $78 \%$ \\
\hline$\geq 2$ & 6 & 21 & & & & \\
\hline$<3$ & 44 & 28 & $13 \%$ & $98 \%$ & $61 \%$ & $80 \%$ \\
\hline$\geq 3$ & 1 & 4 & & & & \\
\hline
\end{tabular}

Note:-NPV indicates negative predictive value; PPV, positive predictive value.

${ }^{a} A$ threshold of 2 has very good overall diagnostic performance.

\section{ROC Curve}

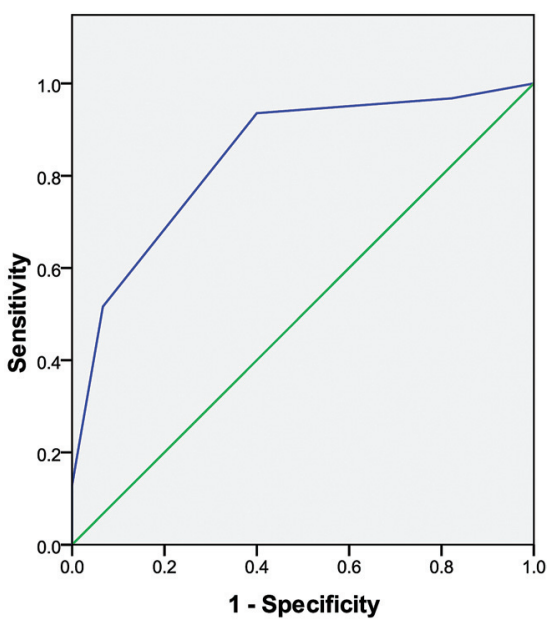

FIG 4. Receiver operating characteristic analysis curve for the scoring system, which showed an area under the curve of 0.841 .

is present in fistulous lesions) or to a reduced outflow. The reduced outflow can be further subdivided into a functional and an anatomic obstruction, with the latter being related often to secondarily acquired venous stenosis. The functional outflow obstruction is related to a shared venous outflow of normal brain and the BAVM. Due to arterialization of the draining vein, drainage of normal brain tissue will be impaired and the amount of tissue impaired by the arterialization will depend on the length of the course of the draining veins over unaffected brain. Venous congestion may be accompanied by a cognitive decline or seizures. ${ }^{13-17,27}$ From an imaging point of view, the pseudophlebitic pattern (increased tortuosity of pial veins) is a direct reflection of venous congestion. ${ }^{19}$ Other signs include delayed venous return of normal brain, venous rerouting, and secondary recruitment of pial superficial veins. Even if signs of venous congestion are not present, a long pial course of the draining vein may indicate that venous drainage stenosis is present over a large area, increasing the risk of venous congestion and subsequent seizures.

Conversely, a short vein that drains almost directly into a dural sinus is unlikely to interfere with the normal brain drainage. The presence of a long pial draining vein may indicate a secondarily acquired phenomenon after progressive stenosis and occlusion of the primary (presumably shorter) draining vein. In our study, the presence of the long draining vein seemed to be the most significant factor associated with seizures (odds ratio of 14.86). This may be similar to resistance of flow for a laminar flow (which may not be the case in BAVMs), which is directly proportional to the length of the vessel and inversely related to the radius of the vessel to the fourth power.

Mass effect is a rare pathomechanism that may result from large venous ectasia or the nidus proper compressing critical structures, and it may lead to seizures, neurologic deficits, and even hydrocephalus. ${ }^{7,28}$ The presence of venous ectasia was also found to be significantly associated with seizures.

These results indicate that the factors reflecting venous congestion are more significantly associated with seizures compared with the factors reflecting high flow and arterial steal. The venous congestion is likely the final common pathway in the ictogenesis in patients with BAVMs.

The decision to surgically treat potentially epileptogenic unruptured BAVMs is still a subject of controversy. ${ }^{29,30}$ Some reports $^{31-33}$ suggest that BAVM surgery allows good control of the seizure disorder. Treatment of such malformations is supposed to decrease the spontaneous risk associated with the malformation and/or cure the symptoms. Better understanding of specific morphologic factors associated with seizure occurrence could further help in understanding the angioarchitectural characteristics responsible for this symptom. These characteristics could then become therapeutic targets for either surgery or embolization.

The proposed scoring system is a potential first step in this direction because it may be helpful in predicting a higher risk of developing seizures in a patient with BAVM on the basis of the individual BAVM angioarchitecture which may, once validated in a prospective fashion on a different, larger population of patients, also guide therapeutic decision-making. Our study identifies certain key predictors (pial long draining vein, venous outflow stenosis, and location) and examines them in combination, which can guide future studies.

Limitations. This was a retrospective study with a small sample size with its associated problems concerning statistical significance. Exclusion of a number of patients due to nonavailability of complete angiograms in the retrospective analysis of our data base makes the selection bias inevitable. In addition, we did not attempt to further subclassify our patients with regard to the type of seizures, frequency of seizures, and drug response and seizure control, to avoid even smaller sample sizes, leading subsequently to lowered statistical significance. Our study is far beyond addressing the complex pathophysiology of inducing a single seizure (ictogenesis) versus the pathomechanisms leading to recurrent seizures (epileptogenesis). However, we hope that our findings will contribute to the puzzle of both conditions going along with a lowered seizure threshold.

\section{CONCLUSIONS}

This study highlights various morphologic and hemodynamic characteristics of unruptured BAVMs that were associated with presentation with seizures. This may help in understanding the 
pathomechanism of seizure occurrence in this patient population and may thereby act as a therapeutic target for either surgery or embolization. The proposed scoring system may help us in predicting seizures in incidentally found BAVMs on the basis of their angioarchitectural features.

Disclosures: Bernhard Pohlmann-Eden—UNRELATED: Consultancy: UCB Pharma; ${ }^{*}$ Payment for Lectures (including service on Speakers Bureaus): UCB Pharma. *Money paid to the institution.

\section{REFERENCES}

1. Stieg P, Batjer HH, Samson D. Intracranial Arteriovenous Malformations. New York: Informa Healthcare USA; 2007:73-79, 215-37

2. Turjman F, Massoud TF, Sayre JW, et al. Epilepsy associated with cerebral arteriovenous malformations: a multivariate analysis of angio-architectural characteristics. AJNR Am J Neuroradiol 1995;16:345-50

3. Al-Shahi R, Warlow CA. Systematic review of the frequency and prognosis of arteriovenous malformations of the brain in adults. Brain 2001;124(pt 10):1900-26

4. Stein BM, Wolpert SM. Arteriovenous malformations of the brain. I. Current concepts and treatment. Arch Neurol 1980;37:1-5

5. Wilkins RH. Natural history of intracranial vascular malformations: a review. Neurosurgery 1985;16:421-30

6. Hoh BL, Chapman PH, Loeffler JS, et al. Results of multimodality treatment for 141 patients with brain arteriovenous malformations and seizures: factors associated with seizure incidence and seizure outcomes. Neurosurgery 2002;51:303-09

7. Hacein-Bey L, Nour R, Pile-Spellman J, et al. Adaptive changes of autoregulation in chronic cerebral hypotension with arteriovenous malformations: an acetazolamide-enhanced single-photon emission CT study. AJNR Am J Neuroradiol 1995;16:1865-74

8. Spetzler RF, Hargraves RW, McCormick PW, et al. Relationship of perfusion pressure and size to risk of hemorrhage from arteriovenous malformations. J Neurosurg 1992;76:918-23

9. Norris JS, Valiante TA, Wallace MC, et al. A simple relationship between radiological arteriovenous malformation hemodynamics and clinical presentation: a prospective, blinded analysis of 31 cases. J Neurosurg 1999;90:673-79

10. Taylor CL, Selman WR, Ratcheson RA. Steal affecting the central nervous system. Neurosurgery 2002;50:679-88

11. Mast H, Mohr JP, Osipov A, et al. 'Steal' is an unestablished mechanism for the clinical presentation of cerebral arteriovenous malformations. Stroke 1995;26:1215-20

12. Mast H, Mohr JP, Thompson JL, et al. Transcranial Doppler ultrasonography in cerebral arteriovenous malformations: diagnostic sensitivity and association of flow velocity with spontaneous hemorrhage and focal neurological deficit. Stroke 1995;26:1024-27

13. Kosnik EJ, Hunt WE, Miller CA. Dural arteriovenous malformations. J Neurosurg 1974;40:322-29

14. Lasjaunias $\mathrm{P}$, Chiu M, ter Brugge $\mathrm{K}$, et al. Neurological manifestations of intracranial dural arteriovenous malformations. J Neurosurg 1986;64:724-30

15. Hurst RW, Hackney DB, Goldberg HI, et al. Reversible arterio- venous malformation-induced venous hypertension as a cause of neurological deficits. Neurosurgery 1992;30:422-25

16. Krings T, Hans FJ, Geibprasert S, et al. Partial “targeted” embolization of brain arteriovenous malformations. Eur Radiol 2010;20: 2723-31

17. Fierstra J, Conklin J, Krings T, et al. Impaired peri-nidal cerebrovascular reserve in seizure patients with brain arteriovenous malformations. Brain 2011;134(pt 1):100-09

18. Santos ML, Demartini Júnior Z, Matos LA, et al. Angio-architecture and clinical presentation of brain arteriovenous malformations. Arq Neuropsiquiatr 2009;67:316-21

19. Willinsky R, Goyal M, terBrugge K, et al. Tortuous, engorged pial veins in intracranial dural arteriovenous fistulas: correlations with presentation, location, and MR findings in 122 patients. AJNR AmJ Neuroradiol 1999;20:1031-36

20. Marks MP, Lane B, Steinberg GK, et al. Hemorrhage in intracerebral arteriovenous malformations: angiographic determinants. Radiology 1990;176:807-13

21. Viñuela F, Drake CG, Fox AJ, et al. Giant intracranial varices secondary to high-flow arteriovenous fistulae. J Neurosurg 1987;66: 198-203

22. Peduzzi P, Concato J, Kemper E, et al. A simulation study of the number of events per variable in logistic regression analysis. J Clin Epidemiol 1996;49:1373-79

23. Crawford PM, West CR, Chadwick DW, et al. Arteriovenous malformations of the brain: natural history in unoperated patients. $\mathrm{JNeu}$ rol Neurosurg Psychiatry 1986;49:1-10

24. Lasjaunias PL, Landrieu P, Rodesch G, et al. Cerebral proliferative angiopathy: clinical and angiographic description of an entity different from cerebral AVMs. Stroke 2008;39:878-85

25. Shankar JJ, Lum C. Whole brain CT perfusion in cases of cerebral arterio-venous malformation. In: Proceedings of the Annual Meeting of the World Federation of Interventional Therapeutic Neuroradiology, Montreal, Quebec, Canada. June 29-July 3, 2009

26. Guo WY, Wu YT, Wu HM, et al. Toward normal perfusion after radiosurgery: perfusion MR imaging with independent component analysis of brain arteriovenous malformations. AJNR Am J Neuroradiol 2004;25:1636-44

27. Alvarez H, Garcia Monaco R, Rodesch G, et al. Vein of Galen aneurysmal malformations. Neuroimaging Clin N Am 2007;17:189-206

28. Geibprasert S, Pereira V, Krings T, et al. Hydrocephalus in unruptured brain arteriovenous malformations: pathomechanical considerations, therapeutic implications and clinical course. J Neurosurg 2009;110:500-07

29. Aminoff M. Treatment of unruptured cerebral arteriovenous malformations. Neurology 1987;37:815-19

30. Heros RC, Tu YK. Is surgical therapy needed for unruptured arteriovenous malformations? Neurology 1987;37:279-86

31. Piepgras DG, Sundt TM Jr, Ragoowansi AT, et al. Seizure outcome in patients with surgically treated cerebral arteriovenous malformations. J Neurosurg 1993;78:5-11

32. Yeh HS, Kashiwagi S, Tew JM Jr, et al. Surgical management of epilepsy associated with cerebral arteriovenous malformations. $\mathrm{J} \mathrm{Neu}$ rosurg 1990;72:216-23

33. Lv X, Li Y, Jiiang C, et al. Brain arteriovenous malformations and endovascular treatment: effect on seizures. Interv Neuroradiol 2010 ; $16: 39-45$ 